\title{
Anapole dark matter quantum mechanics
}

\author{
J. Gamboa, ${ }^{1, *}$ F. Méndez, ${ }^{1, \dagger}$ and N. Tapia ${ }^{2, \$}$ \\ ${ }^{1}$ Departamento de Física, Universidad de Santiago de Chile, Casilla 307, Santiago, Chile \\ ${ }^{2}$ Department of Physics, Virginia Tech, Blacksburg, Virginia 24061, USA
}

(Received 8 October 2019; published 23 January 2020)

\begin{abstract}
The dynamics of an anapole seen as dark matter at low energies is studied by solving the SchrödingerPauli equation in a potential involving the Dirac delta and its derivatives in three dimensions. This is an interesting mathematical problem that, as far as we know, has not been previously discussed. We show how bound states emerge in this approach, and the scattering problem is formulated (and solved) directly. The total cross section is in full agreement with independent calculations in the standard model.
\end{abstract}

DOI: 10.1103/PhysRevD.101.015013

\section{INTRODUCTION}

In the context of dark matter, it is widely believed that some discrete symmetries are not conserved, in the same way that in the visible sector, for example, the parity is broken in the weak interactions. In both cases-dark and visible matter-if parity is violated, an anapole term emerges as the result of the interaction between the spin of the fermions and an electromagnetic source.

This last fact is obtained taking the nonrelativistic limit of

$$
\mathcal{L}=\bar{\chi}(i \not \partial-\bar{m}) \chi-\frac{g}{M^{2}} \bar{\chi} \gamma_{5} \gamma_{\mu} \chi \partial_{\nu} F^{\mu \nu}
$$

where $M$ is a mass scale, $\bar{m}$ is the mass of fermions $\chi$ (electrically neutral), $\gamma^{\mu}$ are the Dirac matrices (with $\mu=0, \ldots, 3), F^{\mu \nu}$ is the electromagnetic strength tensor, and $g$ is the dimensionless coupling constant.

The interaction term in the Lagrangian (1) was proposed by Zeldovich [1] 60 years ago as a way to produce photons by neutral particles such as neutrinos and extending the ideas of parity violation previously proposed by Lee and Yang [2].

This term was named by Zeldovich himself as an anapole interaction, because it is the natural extension of the multipolar expansion for truly neutral interactions (for a review, see [3]).

\footnotetext{
*jorge.gamboa@usach.cl

fernando.mendez@usach.cl

*natalia.tapia@usach.cl
}

Published by the American Physical Society under the terms of the Creative Commons Attribution 4.0 International license. Further distribution of this work must maintain attribution to the author(s) and the published article's title, journal citation, and DOI. Funded by SCOAP.
Even more, in the context of Lee and Yang's parity violation [2], Zeldovich pointed out [1] that the process $\nu \bar{\nu}$ could also produce a virtual photon and, therefore, violate parity, assuming an effective vertex as in Fig. 1. Since the incoming particles are neutral, the interaction is described through the anapole which is, technically, the analog of the next to quadrupolar term in the multipolar expansion of $\frac{1}{|\mathbf{x}-\mathbf{y}|}$ in electrodynamics. However, although this effect is very weak, it is measurable, and its detection was announced in 1997 [4] for the transition $6 S$ to $7 S$ in cesium atoms (see also [5]).

The idea of anapoles has been used recently by several authors [6] and mainly by Ho and Scherrer [7], who have proposed that the anapole could be considered a form of dark matter.

In this paper, we study some properties of this anapole interaction from the point of view of quantum mechanics, and we show the emergence of nontrivial properties such as the formation of bound states and a notorious simplicity in scattering processes.

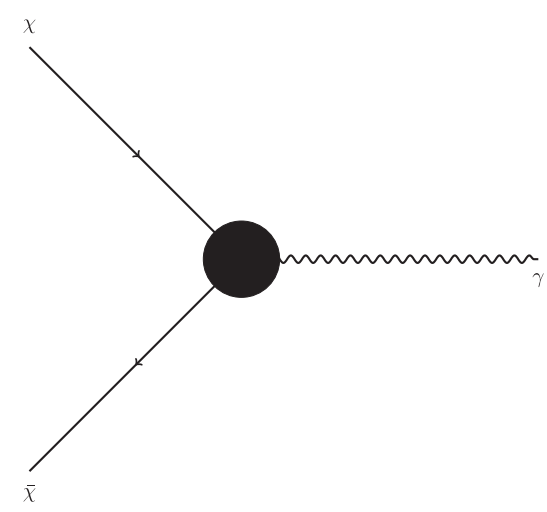

FIG. 1. This is a process of annihilation of two fermions in a photon $\gamma$. The black box is an effective vertex. 
The paper is organized as follows: In Sec. II, we discuss the nonrelativistic anapole, and we formulate the problem in general; in Sec. III, we discuss the bound state and scattering problem in three dimensions, and we provide an exact solution of the Schrödinger-Pauli equation; in Sec. IV, we discuss our results. Two Appendixes of technical issues are included.

\section{NONRELATIVISTIC ANAPOLE}

The nonrelativistic limit [8] of the Hamiltonian (1) is

$$
\begin{aligned}
H & =\frac{1}{2 m} \mathbf{p}^{2}-\frac{g}{M^{2}} \mathbf{S} \cdot \mathbf{J}+\mathcal{O}\left(c^{2}\right) \\
& =\frac{1}{2 m} \mathbf{p}^{2}+H_{\text {anap }}+\mathcal{O}\left(c^{2}\right),
\end{aligned}
$$

where the first term is the kinetic energy of the leptons with three-momentum $\mathbf{p}$ and $H_{\text {anap }}=-\frac{g}{M^{2}} \mathbf{S} \cdot \mathbf{J}$ is the Hamiltonian describing the anapole interaction, with $\mathbf{S}=\frac{1}{2} \boldsymbol{\sigma}$ its spin and $\mathbf{J}$ the electromagnetic source (coming from Ampere's law).

In the case of dark matter, the above issue is a little bit more involved, because the interaction between visible and dark matter is indirect and the concept of kinetic mixing is necessary [9].

The interaction between the dark fermions and the gauge fields is produced by the diagonalization of the kinetic mixing implying that, instead of (2), for the case of dark matter one has to consider

$$
\begin{aligned}
H & =\frac{1}{2 m} \mathbf{p}^{2}-\frac{g^{\prime}}{M^{2}} \mathbf{S}_{\mathrm{dark}} \cdot \mathbf{J}+\mathcal{O}\left(c^{2}\right) \\
& =\frac{1}{2 m} \mathbf{p}^{2}+H_{\mathrm{dark}}+\mathcal{O}\left(c^{2}\right),
\end{aligned}
$$

where, now, $g^{\prime}=\xi g$ is an effective coupling constant, $\xi \ll 1$ is the kinetic mixing parameter, and the anapole describes the interaction between dark matter and the electromagnetic source.

The change $g \rightarrow g^{\prime}$ is a nontrivial consequence of the diagonalization of the kinetic mixing and the gauge group enlargement $U(1)$ to $U(1) \times U(1)$.

Then, as $\mathbf{J}$ is a current density of particles of the standard model, we can assume that the particles are millicharged and Ohm's law implies $\mathbf{J}=\rho \mathbf{v}$, where $\mathbf{v}$ is the velocity of the charge carriers moving in a volume of $V$ with the charge density $\rho$.

As the charge carriers are point particles, $\rho \sim \delta(\mathbf{x})$, and the classical anapole Hamiltonian can be written as

$$
H_{\text {anap }}=-\frac{g^{\prime}}{M^{2} m} \boldsymbol{\sigma} \cdot \mathbf{p} \delta(\mathbf{x}),
$$

which is an "electromagnetic" interaction $\mathbf{J} \cdot \mathbf{A}$, where $\mathbf{A}$ is formally identified with $\sigma \delta(\mathbf{x})$, which is an AharonovBohm-like effect. In other words, we have a toroidal cylinder with a magnetic field confined inside in a similar way to the Tonomura experiments for the Aharonov-Bohm effect [10].

Then, in our nonrelativistic quantum mechanics model, particles with momentum $\mathbf{p}$ interact with dark matter through an anapole term, giving rise to the total Hamiltonian operator

$$
\hat{H}=\frac{1}{2 m} \mathbf{p}^{2}-\frac{g^{\prime}}{2 M^{2} m}(\delta(\mathbf{x}) \boldsymbol{\sigma} \cdot \mathbf{p}+\boldsymbol{\sigma} \cdot \mathbf{p} \delta(\mathbf{x})),
$$

and the solution to this problem will provide a complete quantum mechanical picture, including bound and scattering states.

In order to solve the eigenvalue problem, let us start considering the following Schrödinger-Pauli equation:

$$
\left(\boldsymbol{\nabla}^{2}+k^{2}\right) \psi(\mathbf{x})=-\frac{i g^{\prime}}{M^{2}}[\delta(\mathbf{x}) \boldsymbol{\sigma} \cdot \nabla \psi(\mathbf{x})+\boldsymbol{\sigma} \cdot \boldsymbol{\nabla} \delta(\mathbf{x}) \psi(\mathbf{x})],
$$

where $k^{2}=2 m E$.

The previous equation (6) can be recast as an integral equation ${ }^{1}$ :

$$
\begin{aligned}
\psi(\mathbf{x})= & \psi_{0}(\mathbf{x})-\frac{i g^{\prime}}{M^{2}} \int d^{D} x^{\prime} G\left[\mathbf{x}, \mathbf{x}^{\prime}\right]\left[\delta\left(\mathbf{x}^{\prime}\right) \boldsymbol{\sigma} \cdot \nabla^{\prime} \psi\left(\mathbf{x}^{\prime}\right)\right. \\
& \left.+\boldsymbol{\sigma} \cdot \nabla^{\prime} \delta\left(\mathbf{x}^{\prime}\right) \psi\left(\mathbf{x}^{\prime}\right)\right] \\
= & \psi_{0}(\mathbf{x})-\frac{i g^{\prime}}{M^{2}}(G[\mathbf{x}, 0] \boldsymbol{\sigma} \cdot \boldsymbol{\nabla} \psi(0)-\boldsymbol{\sigma} \cdot \boldsymbol{\nabla} G[\mathbf{x}, 0] \psi(0)),
\end{aligned}
$$

with $\psi_{0}(\mathbf{x})=A e^{i \mathbf{k} \cdot \mathbf{x}}$, the homogeneous solution of the operator $\boldsymbol{\nabla}^{2}+k^{2}$, and the Green function $G\left[\mathbf{x}, \mathbf{x}^{\prime}\right]$ given by

$$
\begin{aligned}
G\left[\mathbf{x}, \mathbf{x}^{\prime}\right] & =\int \frac{d^{D} p}{(2 \pi)^{D}} \frac{e^{i \mathbf{p} \cdot\left(\mathbf{x}-\mathbf{x}^{\prime}\right)}}{\mathbf{p}^{2}+k^{2}} \\
& =\left(\frac{\left|\mathbf{x}-\mathbf{x}^{\prime}\right|}{2|k|}\right)^{1-(D / 2)} K_{1-(D / 2)}\left(|k|\left|\mathbf{x}-\mathbf{x}^{\prime}\right|\right) .
\end{aligned}
$$

An instructive example is to consider the onedimensional case, where the previous solution reduces to

$\psi(x)=\psi_{0}(x)-\frac{i g^{\prime}}{M^{2}}\left[G[x, 0] \psi^{\prime}(0)-G^{\prime}[x, 0] \psi(0)\right]$.

It is interesting to determine the bound states of this problem. Then $\psi_{0}=0$, and we evaluate the previous expression at $x=0$, that is,

\footnotetext{
${ }^{1}$ For convenience, we will do the calculation in $D$ dimensions.
} 


$$
\left(1-\frac{i g^{\prime}}{M^{2}} G^{\prime}[0,0]\right) \psi(0)=-\frac{i g^{\prime}}{M^{2}} G[0,0] \psi^{\prime}(0) .
$$

We impose Robin's boundary condition

$$
\psi^{\prime}(0)=\gamma \psi(0),
$$

where $\gamma \in \operatorname{Re}$ is the parameter that defines the self-adjoint extension of the Hamiltonian, to find

$$
\left(1-\frac{i g^{\prime}}{M^{2}} G^{\prime}[0,0]\right)=-\frac{i g^{\prime} \gamma G[0,0]}{M^{2}} .
$$

The functions $G[0,0]$ and $G^{\prime}[0,0]$, from (9), are, respectively,

$$
G[0,0]=-\frac{i \pi}{k}, \quad G^{\prime}[0,0]=0,
$$

therefore

$$
E=\frac{k^{2}}{2 m}=\frac{g^{\prime 2} \gamma^{2} \pi^{2}}{2 m M^{4}}>0,
$$

and therefore there are not bound states in the onedimensional case.

\section{THREE-DIMENSIONAL NONRELATIVISTIC ANAPOLE; BOUND AND SCATTERING STATES}

The three-dimensional case is more complicated and has important differences with the previous one-dimensional example, such as the existence of the bound state and renormalization of the coupling constant as a consequence of the three-dimensional scale invariance $[11,12] .{ }^{2}$

Then for bound states we put $\psi_{0}=0$ and (7) becomes

$$
\begin{aligned}
\psi(\mathbf{x})= & -\frac{i g^{\prime}}{2 M^{2}} \int d^{3} x^{\prime} G\left[\mathbf{x}, \mathbf{x}^{\prime}\right] \delta\left(\mathbf{x}^{\prime}\right) \boldsymbol{\sigma} \cdot \nabla^{\prime} \psi\left(\mathbf{x}^{\prime}\right) \\
& -\frac{i g^{\prime}}{2 M^{2}} \int d^{3} x^{\prime} G\left[\mathbf{x}, \mathbf{x}^{\prime}\right] \boldsymbol{\sigma} \cdot \nabla^{\prime}\left(\delta\left(\mathbf{x}^{\prime}\right) \psi\left(\mathbf{x}^{\prime}\right)\right) .
\end{aligned}
$$

We are interested in the case $\mathbf{x}=0=\mathbf{x}^{\prime}$, and due to the spherical symmetry and the explicit form of the Green function one gets $\partial_{r} G \rightarrow 0$.

Therefore, we write (16) as follows:

$$
\psi(\mathbf{x})=-\frac{l g^{\prime}}{M^{2}} \int G\left(\mathbf{x}, \mathbf{x}^{\prime}\right)\left[\delta\left(\mathbf{x}^{\prime}\right)\left(\boldsymbol{\sigma} \cdot \nabla^{\prime}\right)\right] \psi\left(\mathbf{x}^{\prime}\right) d^{3} x^{\prime}
$$

Integral (17) can be done straightforwardly. To do that, we write explicitly the spinors and look for solutions with the form

\footnotetext{
${ }^{2}$ For continuity of the presentation of our results, some technical and notation aspects are relegated to Appendixes A and $\mathrm{B}$.
}

$$
\psi(\mathbf{x})=\Phi_{+} \xi^{+}+\Phi_{-} \xi^{-},
$$

where $\Phi^{ \pm}$are functions of $r$, while spinors carry the angular dependence.

It is interesting to note that in usual cases the radial functions are equals $\left(\Phi_{+}=\Phi_{-}\right)$, but in our case the interaction changes the orbital angular momentum states according to (A9), which supports our choice, and then the state is a superposition of the two different orbital angular momentum $\ell=j \pm \frac{1}{2}$ for a fixed total angular momentum $j$.

Now we use the explicit form of the Green function and integrate (17) for the solution in (18), and we evaluate in $\mathbf{x}=0$. One gets

$$
\left[\Phi_{+}(0)-\frac{\imath g^{\prime}}{M^{2}} G_{\Lambda} \Phi_{-}^{\prime}(0)\right] \xi^{+}=\left[\Phi_{-}(0)-\frac{\imath g^{\prime}}{M^{2}} G_{\Lambda} \Phi_{+}^{\prime}\right] \xi^{-}
$$

where $g^{\prime}$ has been rescaled through $g^{\prime} \rightarrow g^{\prime}(1+\theta(0))$ as a consequence of scale invariance $[11,12]$.

The Green function $G_{\Lambda}$, instead, has been regularized through an ultraviolet cutoff $\Lambda$ for $\mathbf{x}, \mathbf{x}^{\prime} \rightarrow 0$ (see Appendix B).

The previous equations can be recast as

$$
\left(\begin{array}{l}
\Phi_{+} \\
\Phi_{-}
\end{array}\right)_{0}=\frac{l g^{\prime} G_{\Lambda}}{M^{2}}\left(\begin{array}{ll}
0 & 1 \\
1 & 0
\end{array}\right)\left(\begin{array}{l}
\Phi_{+}^{\prime} \\
\Phi_{-}^{\prime}
\end{array}\right)_{0}
$$

where subscript 0 stand for $\mathbf{x}=0$.

In order to find the bound states, we posit a generalization of the Robin's boundary conditions, as follows:

$$
\left(\begin{array}{l}
\Phi_{+}^{\prime} \\
\Phi_{-}^{\prime}
\end{array}\right)_{0}=\mathbb{G}\left(\begin{array}{l}
\Phi_{+} \\
\Phi_{-}
\end{array}\right)_{0}
$$

with $\mathbb{G}$ a $2 \times 2$ matrix with dimensions of mass.

With this choice, condition (21) reads

$$
\left(\mathbb{I}-\frac{\imath g^{\prime} G_{\Lambda}}{M^{2}} \sigma_{1} \mathbb{G}\right)\left(\begin{array}{l}
\Phi_{+} \\
\Phi_{-}
\end{array}\right)_{0}=0
$$

and the condition for bound sates is

$$
\operatorname{det}\left(\mathbb{I}-\frac{l g^{\prime} G_{\Lambda}}{M^{2}} \sigma_{1} \mathbb{G}\right)=0 .
$$

We look for imaginary solutions for $k$, since then $k^{2}<0$, corresponding to negative energy states. For the Green function (see Appendix B)

$$
G_{\Lambda}=\frac{k}{4 \pi \imath}-\Lambda
$$

we choose 


$$
\mathbb{G}=\kappa\left(\begin{array}{cc}
\alpha & -\imath \beta \\
\imath \beta & -\alpha
\end{array}\right)
$$

with $\{\alpha, \beta\} \in \operatorname{Re}, \alpha^{2}+\beta^{2}=1$, and $\kappa$, a constant with dimensions of mass. Note that $\mathbb{G}^{\dagger} \mathbb{G}=\kappa^{2} \mathbb{I}$. By doing this, we get

$$
k_{ \pm}=4 \pi \imath\left(\Lambda \pm \frac{M^{2}}{\kappa g^{\prime}}\right)
$$

and, therefore, the energy of the bound states turns out to be

$$
\left|E_{ \pm}\right|=\frac{8 \pi^{2}}{m}\left(\Lambda \pm \frac{M^{2}}{\kappa g^{\prime}}\right)^{2}
$$

implying that, for this particular choice of Robin's boundary conditions, there exist bound states for $\kappa \in$ Re. Note also that, for the particular choice of $\kappa_{\mp}=\mp \frac{M^{2}}{\Lambda g^{\prime}}$, the bound states have zero energy.

For scattering processes, a similar behavior is verified. Indeed, the problem can be formulated as follows: First, we rewrite (8) iteratively:

$$
\begin{aligned}
\psi(\mathbf{x})= & \psi_{0}(\mathbf{x})-\frac{i g^{\prime}}{M^{2}} G[\mathbf{x}, 0]\left(\boldsymbol{\sigma} \cdot \nabla \psi_{0}(0)\right) \\
& -(\boldsymbol{\sigma} \cdot \boldsymbol{\nabla} G[\mathbf{x}, 0]) \psi_{0}(0)+\cdots
\end{aligned}
$$

However, the last term in the rhs of Eq. (26) is energetically less relevant than the first one, and we can write the last equation as

$$
\psi(\mathbf{x})=\psi_{0}(\mathbf{x})-\frac{i g^{\prime}}{M^{2}} G[\mathbf{x}, 0]\left(\boldsymbol{\sigma} \cdot \nabla \psi_{0}(0)\right)+\cdots
$$

The appropriate Green function for the boundary conditions of the scattering problem is

$$
G[\mathbf{x}, 0]=-\frac{1}{4 \pi} \frac{e^{i k r}}{r},
$$

and therefore scattering state (27) is

$$
\psi(\mathbf{x})=\varphi_{0}(\mathbf{x})+A \frac{e^{i k r}}{r},
$$

where, formally,

$$
A=-\frac{i g^{\prime}}{4 \pi M^{2}}\left(\boldsymbol{\sigma} \cdot \nabla \psi_{0}(0)\right)
$$

is our definition of the scattering amplitude.

We prepare the initial state as $\psi_{0}=\xi^{+} \psi_{+}+\xi^{-} \psi_{-}$(in principle, we can take $\psi_{+}=\psi_{-}=e^{\imath k z}$ ), and we get

$$
\left(\boldsymbol{\sigma} \cdot \nabla \psi_{0}\right)_{\mathbf{x}=0}=-\left(\boldsymbol{\xi}^{-} \partial_{r} \psi_{+}+\boldsymbol{\xi}^{+} \partial_{r} \psi_{-}\right)_{\mathbf{x}=0}
$$

and, therefore, the total scattering amplitude $\sigma_{\mathrm{TOT}}=A^{\dagger} A$ turns out to be

$$
\sigma_{\mathrm{TOT}}=\frac{g^{\prime 2}}{16 \pi^{2} M^{4}}\left(\left|\partial_{r} \psi_{+}(0)\right|^{2}+\left|\partial_{r} \psi_{-}(0)\right|^{2}\right) .
$$

We impose now Robin's boundary condition (21) with $\mathbb{G}^{\dagger} \mathbb{G}=\kappa^{2} \mathbb{I}$, and therefore the total cross section is

$$
\sigma_{\mathrm{TOT}}=\frac{g^{\prime 2} \kappa^{2}}{16 \pi^{2} M^{4}}\left(\left|\psi_{+}(0)\right|^{2}+\left|\psi_{-}(0)\right|^{2}\right) \text {. }
$$

We note that, up to the normalization factor $\left(\left|\psi_{+}(0)\right|^{2}+\left|\psi_{-}(0)\right|^{2}\right)$, this result is in full agreement with independent calculations reported in Ref. [13] if we interpret $\kappa$ as the mass of the dark matter nucleon.

From the above results, we could conclude that having sufficiently reliable bounds for $\Lambda$ and $M$ could be argued for observability (or not) of anapole dark matter.

A careful analysis of the xenon-100 data [14] and the bounds for hidden photons from Ref. [15] could help clarify these issues.

\section{FINAL COMMENTS}

As final comments, we point out the following: (a) The anapole observables in scattering processes are dependent only on the mass scale and coupling constant that appear in the Zeldovich term-therefore, the number of adjustable parameters needed to extract bounds is minimal, and this is an advantage with respect to other approaches; (b) the approach proposed here not only provides a systematic way of carrying out parity violation effects as a consequence of the standard model [16], but can also be considered as a starting point to study possible corrections associated with dark matter and its interactions.

\section{ACKNOWLEDGMENTS}

We thank Manuel Asorey, Paola Arias, and Jose G. Esteve for discussions. This work was supported by Dicyt 041831GR (J. G.) and 041931MF (F. M.). One of us (J. G.) thanks the DESY theory group and the Alexander von Humboldt Foundation for the hospitality and support.

\section{APPENDIX A: THE EIGENSPINORS AND THE EQUATION OF MOTION}

In order to build the solution of the equation of motion, let us review some facts related to the spinor factorization [17].

Consider the Hamiltonian

$$
\mathcal{H}=\mathbf{L}^{2}-\mathbf{J}^{2}-\frac{1}{4}
$$

with the vector operators $\mathbf{L}=\mathbf{r} \times \mathbf{p}$, the orbital angular momentum, and $\mathbf{J}=\mathbf{L}+\mathbf{S}$, the total angular momentum. 
The spin operator is $\mathbf{S}$, and we take $\mathbf{S}=\frac{1}{2} \boldsymbol{\sigma}$, with $\boldsymbol{\sigma}$ the Pauli matrices.

It is direct to prove that

$$
\mathcal{H}=-\boldsymbol{\sigma} \cdot \mathbf{L}-1,
$$

which will be useful in what follows. On the other hand, the eigenvalue problem

$$
\mathcal{H}|k\rangle=\kappa|k\rangle
$$

has the solution

$$
\kappa=\ldots,-2,-1,1,2, \ldots
$$

with $\kappa \neq 0$.

For a given $\kappa$, the eigenvalues of $\mathbf{L}^{2}$ and $\mathbf{J}^{2}[\ell(\ell+1)$ and $j(j+1)$, respectively] satisfy

$$
\ell=|\kappa|+\frac{1}{2}(\operatorname{sgn}(\kappa)-1), \quad j=|\kappa|-\frac{1}{2} .
$$

In the usual notation we have, therefore,

$$
j=\frac{1}{2}, \frac{3}{2}, \frac{5}{2}, \frac{7}{2} \ldots,
$$

with $j=\ell \pm \frac{1}{2}$ and the projection $m_{j}$ (eigenvalue of $J_{3}$ ) is given by the $2 j+1$ values:

$$
m_{j}=-j,-j+1,-j+2, \ldots, j-2, j-1, j .
$$

Eigenfunctions of the commuting set of operators $\left\{\mathbf{L}^{2}, \mathbf{J}^{2}, J_{3}\right\}$ are the spinor spherical harmonics (Pauli spinors)

$$
\begin{aligned}
& \xi_{\ell, j=\ell-(1 / 2)}^{m_{j}}=\left(\begin{array}{c}
-\sqrt{\frac{\ell-m_{j}+\frac{1}{2}}{2 \ell+1}} Y_{\ell}^{m_{j}-(1 / 2)} \\
\sqrt{\frac{\ell+m_{j}+\frac{1}{2}}{2 \ell+1}} Y_{\ell}^{m_{j}+(1 / 2)}
\end{array}\right) \quad \text { for } \kappa>0, \\
& \xi_{\ell, j=\ell+(1 / 2)}^{m_{j}}=\left(\begin{array}{c}
\sqrt{\frac{\ell+m_{j}+\frac{1}{2}}{2 \ell+1}} Y_{\ell}^{m_{j}-(1 / 2)} \\
\sqrt{\frac{\ell-m_{j}+\frac{1}{2}}{2 \ell+1}} Y_{\ell}^{m_{j}+(1 / 2)}
\end{array}\right) \quad \text { for } \kappa<0 .
\end{aligned}
$$

For a fixed total angular momentum $j$, previous spinors correspond to two angular momentum $\ell$, namely, $\ell=j \pm \frac{1}{2}$.

Let us introduce the notation $\xi^{+}$for the spinor in Eq. (A7) and $\xi^{-}$for the one in Eq. (A8) omitting labels $\ell, j$, and $m_{j}$. The following relation holds:

$$
(\hat{\mathbf{r}} \cdot \boldsymbol{\sigma}) \boldsymbol{\xi}^{ \pm}=-\xi^{\mp} .
$$

This relation is useful, because the interaction term can be written in terms of previous operator and $\mathcal{H}$ only. Indeed, from $\mathbf{L}=-\imath \mathbf{r} \times \mathbf{\nabla}$, the following identity holds:

$$
\boldsymbol{\nabla}=\hat{\mathbf{r}}(\hat{\mathbf{r}} \cdot \boldsymbol{\nabla})+\frac{l}{r^{2}} \mathbf{r} \times \mathbf{L},
$$

and therefore

$$
\boldsymbol{\sigma} \cdot \boldsymbol{\nabla}=(\hat{\mathbf{r}} \cdot \boldsymbol{\sigma})(\hat{\mathbf{r}} \cdot \boldsymbol{\nabla})+\frac{l}{r^{2}} \boldsymbol{\sigma} \cdot(\mathbf{r} \times \mathbf{L}) .
$$

The last term in the previous expression can be written as $\boldsymbol{\sigma} \cdot(\mathbf{r} \times \mathbf{L})=\imath(\mathbf{r} \cdot \boldsymbol{\sigma})(\boldsymbol{\sigma} \cdot \mathbf{L})$, once the relation $\sigma_{i} \sigma_{j}=$ $\delta_{i j}+\imath \epsilon_{i j k} \sigma_{k}$ is used.

Then we get for the interaction term

$$
\boldsymbol{\sigma} \cdot \boldsymbol{\nabla}=(\hat{\mathbf{r}} \cdot \boldsymbol{\sigma})\left[(\hat{\mathbf{r}} \cdot \boldsymbol{\nabla})-\frac{1}{r}(\boldsymbol{\sigma} \cdot \mathbf{L})\right] .
$$

Finally, we can use Eq. (A2) in order to replace the last term by $\mathcal{H}+1$. Since $(\hat{\mathbf{r}} \cdot \boldsymbol{\nabla})=\partial_{r}$, it is possible to write the interaction term as follows:

$$
\boldsymbol{\sigma} \cdot \boldsymbol{\nabla}=(\hat{\mathbf{r}} \cdot \boldsymbol{\sigma})\left[\partial_{r}+\frac{1}{r}(\mathcal{H}+1)\right] .
$$

The action of this operator on the spinors $\xi^{ \pm}$is obtained from Eq. (A9):

$$
\boldsymbol{\sigma} \cdot \boldsymbol{\nabla} \boldsymbol{\xi}^{ \pm}=-\boldsymbol{\xi}^{\mp}\left[\partial_{r}+\frac{1}{r}(1 \pm|\kappa|)\right] .
$$

Then, the interaction terms changes the orbital angular momentum $\ell$ when the total angular momentum $j$ is fixed.

In particular, for a wave function with the form

$$
\psi^{ \pm}(\mathbf{x})=\Phi^{ \pm}(r) \boldsymbol{\xi}^{ \pm}(\theta, \varphi),
$$

we get

$$
\boldsymbol{\sigma} \cdot \boldsymbol{\nabla} \psi^{ \pm}(\mathbf{x})=-\boldsymbol{\xi}^{\mp}\left[\partial_{r} \Phi^{ \pm}+\frac{\Phi^{ \pm}}{r}(1 \pm|\kappa|)\right] .
$$

\section{APPENDIX B: THE GREEN FUNCTION}

In this section, we calculate the Green function for the Helmholtz equation. The Green function in this case satisfies

$$
\left(\boldsymbol{\nabla}^{2}+k^{2}\right) G\left(\mathbf{x}, \mathbf{x}^{\prime}\right)=\delta\left(\mathbf{x}-\mathbf{x}^{\prime}\right),
$$

and therefore

$$
G\left(\mathbf{x}, \mathbf{x}^{\prime}\right)=\frac{1}{(2 \pi)^{3}} \int \frac{e^{\imath \mathbf{p} \cdot\left(\mathbf{x}-\mathbf{x}^{\prime}\right)}}{k^{2}-p^{2}} d^{3} p .
$$

By performing the integration on the angular variables, we get 


$$
G\left(\mathbf{x}, \mathbf{x}^{\prime}\right)=\frac{1}{2 \pi^{2}\left|\mathbf{x}-\mathbf{x}^{\prime}\right|} \int_{0}^{\infty} \frac{p}{k^{2}-p^{2}} \sin \left(p\left|\mathbf{x}-\mathbf{x}^{\prime}\right|\right) d p .
$$

The last integral is divergent due to the pole at $p=k$.

We are interested in bound states; therefore, $k^{2}=$ $-2 m|E| \equiv-\epsilon^{2}$, and in such a case, we are interested in the following expression:

$$
I=\frac{1}{\Delta} \int_{0}^{\infty} \frac{p \sin (p \Delta)}{\epsilon^{2}+p^{2}} d p
$$

with $\Delta=\left|\mathbf{x}-\mathbf{x}^{\prime}\right|$. The factor $\Delta^{-1}$ has been introduced for further convenience.

In order to integrate the previous expression, we introduce a cutoff $\Lambda$ and consider, instead,

$$
\begin{aligned}
I_{\Lambda} \equiv & \frac{1}{\Delta} \int_{0}^{\Lambda} \frac{p \sin (p \Delta)}{\epsilon^{2}+p^{2}} d p \\
= & \frac{\imath}{2 \Delta} \sinh (\Delta \epsilon)[\operatorname{Ci}(\Delta(\Lambda-\imath \epsilon))-\operatorname{Ci}(\Delta(\Lambda+\imath \epsilon))+\imath \pi] \\
& -\frac{1}{\Delta} \cosh (\Delta \epsilon)[\operatorname{Si}(\Delta(\Lambda-\imath \epsilon))+\operatorname{Si}(\Delta(\Lambda+\imath \epsilon))]
\end{aligned}
$$

where $\operatorname{Si}(x)$ denotes the sine integral function and $\operatorname{Ci}(x)$ is the cosine integral.

In order to get the behavior of this integral for $\Delta \epsilon \rightarrow 0$ and $\Delta \Lambda \rightarrow 0$, we expand in these dimensionless variables to obtain

$$
I_{\Lambda}=\Lambda-\frac{\pi \epsilon}{2}+\mathcal{O}\left(\Delta^{2} \Lambda^{2}\right)
$$

By replacing this result in the Green function (B3), we get the Green function in the limit $\mathbf{x}, \mathbf{x}^{\prime} \rightarrow 0$ :

$$
\begin{aligned}
G_{\Lambda} & =-\frac{1}{2 \pi^{2}}\left(\Lambda+\frac{l \pi k}{2}\right) \\
& =\frac{k}{4 \pi \imath}-\Lambda,
\end{aligned}
$$

where we have restored $\epsilon=-l k$ for the bound states.
Regarding the derivative of the Green function, it is enough to note that we are interested only in $\Delta=r$, and, therefore, we consider $\partial_{r}$. From Eq. (B4), one obtains

$$
\partial_{r} I_{\Lambda}=\frac{\pi \Delta \epsilon^{3}}{6}-\frac{\Delta \epsilon^{4}}{3 \Lambda}+\mathcal{O}\left(\Delta^{3} \Lambda\right)
$$

and therefore

$$
\partial_{r} G_{\Lambda} \rightarrow 0
$$

for $r \rightarrow 0$.

Finally, let us show a simpler way to obtain the previous results. In this approach, we take the limit $\Delta \rightarrow 0$ before integration, that is,

$$
\begin{aligned}
I_{\Lambda}(\Delta \rightarrow 0) & =\int_{0}^{\Lambda} \frac{p^{2}}{\epsilon^{2}+p^{2}} d p \\
& =\Lambda-\epsilon \arctan \left(\frac{\Lambda}{\epsilon}\right) \\
& =\Lambda-\frac{\pi \epsilon}{2}+\mathcal{O}(\epsilon / \Lambda) .
\end{aligned}
$$

In the limit $\Lambda \rightarrow \infty$, only the first two terms are relevant. Finally (restoring also $\epsilon=-l k$ ), we find

$$
I_{\Lambda}=\Lambda+\frac{\imath \pi k}{2}
$$

which is the same result as before, and, therefore,

$$
G_{\Lambda}=\frac{k}{4 \pi \imath}-\Lambda
$$

The derivative, in this approach, is direct to calculate, since

$$
\partial_{\Delta}\left[\frac{p \sin (p \Delta)}{\Delta\left(\epsilon^{2}+p^{2}\right)}\right]=\frac{p}{\Delta\left(\epsilon^{2}+p^{2}\right)}\left[p \cos (p \Delta)-\frac{\sin (p \Delta)}{\Delta}\right],
$$

and it is direct to prove that in the limit $\Delta \rightarrow 0$ the previous expression vanishes.
[1] Y. B.Zeldovich,ZhETF 33, 1531 (1958) [JETP 6, 1185 (1958)].

[2] T. D. Lee and C. N. Yang, Phys. Rev. 104, 254 (1956).

[3] See e.g., G. Afanasiev and Y. P. Stepanovsky, J. Phys. A 28, 4565 (1995).

[4] C. S. Wood, S. C. Bennett, D. Cho, B. P. Masterson, J. L. Roberts, C. E. Tanner, and C. E. Wieman, Science 275, 1759 (1997).
[5] M. S. Safronova, D. Budker, D. DeMille, D. F. J. Kimball, A. Derevianko, and C. W. Clark, Rev. Mod. Phys. 90, 025008 (2018).

[6] D. C. Latimer, Phys. Rev. D 95, 095023 (2017); S. Kang, S. Scopel, G. Tomar, J. H. Yoon, and P. Gondolo, J. Cosmol. Astropart. Phys. 11 (2018) 040; A. Alves, A. C. O. Santos, and K. Sinha, Phys. Rev. D 97, 055023 (2018); X. Chu, 
J. Pradler, and L. Semmelrock, Phys. Rev. D 99, 015040 (2019).

[7] C. M. Ho and R. J. Scherrer, Phys. Lett. B 722, 341 (2013); Y. Gao, C. M. Ho, and R. J. Scherrer, Phys. Rev. D 89, 045006 (2014).

[8] P. Arias, J. Gamboa, and N. Tapia, Phys. Lett. B 791, 17 (2019).

[9] B. Holdom, Phys. Lett. 166B, 196 (1986).

[10] A. Tonomura, T. Matsuda, R. Suzuki, A. Fukuhara, N. Osakabe, H. Umezaki, J. Endo, K. Shinagawa, Y. Sugita, and H. Fujiwara, Phys. Rev. Lett. 48, 1443 (1982); 56, 792 (1986).

[11] R. Jackiw, Diverse Topics in Theoretical and Mathematical Physics (World Scientific, Singapore, 1994).
[12] H. E. Camblong, L. N. Epele, H. Fanchiotti, and C. A. Garcia Canal, Ann. Phys. (N.Y.) 287, 14 (2001); Phys. Rev. Lett. 85, 1590 (2000).

[13] E. Del Nobile, G. B. Gelmini, P. Gondolo, and J. H. Huh, J. Cosmol. Astropart. Phys. 06 (2014) 002.

[14] J. Angle et al. (XENON Collaboration), Phys. Rev. Lett. 100, 021303 (2008).

[15] I. G. Irastorza and J. Redondo, Prog. Part. Nucl. Phys. 102, 89 (2018).

[16] M. A. Bouchiat and C. C. Bouchiat, Phys. Lett. 48B, 111 (1974); M. A. Bouchiat and C. Bouchiat, J. Phys. II (France) 35, 899 (1974); Rep. Prog. Phys. 60, 1351 (1997).

[17] L. C. Biedenharn and J. D. Louck, Encycl. Math. Appl. 8, 1 (1981). 